Journal of

Synchrotron

Radiation

ISSN 0909-0495

Received 31 January 2013

Accepted 7 April 2013

\section{Thermal deformation of cryogenically cooled silicon crystals under intense $X$-ray beams: measurement and finite-element predictions of the surface shape}

\author{
Lin Zhang, ${ }^{*}$ Manuel Sánchez del Río, Giulio Monaco, Carsten Detlefs, \\ Thomas Roth, $\neq$ Aleksandr I. Chumakov and Pieter Glatzel
}

European Synchrotron Radiation Facility, 6 rue Jules Horowitz, BP 220, Grenoble 38043, France. E-mail: zhang@esrf.fr

\begin{abstract}
X-ray crystal monochromators exposed to white-beam X-rays in thirdgeneration synchrotron light sources are subject to thermal deformations that must be minimized using an adequate cooling system. A new approach was used to measure the crystal shape profile and slope of several cryogenically cooled (liquid nitrogen) silicon monochromators as a function of beam power in situ and under heat load. The method utilizes multiple angular scans across the Bragg peak (rocking curve) at various vertical positions of a narrow-gap slit downstream from the monochromator. When increasing the beam power, the surface of the liquid-nitrogen-cooled silicon crystal deforms from a concave shape at low heat load to a convex shape at high heat load, passing through an approximately flat shape at intermediate heat load. Finite-element analysis is used to calculate the crystal thermal deformations. The simulated crystal profiles and slopes are in excellent agreement with experiments. The parameters used in simulations, such as material properties, absorbed power distribution on the crystal and cooling boundary conditions, are described in detail as they are fundamental for obtaining accurate results.
\end{abstract}

Keywords: silicon crystal; X-ray monochromator; cryogenic cooling; liquid nitrogen; thermal deformation; finite element; heat load experiment; rocking curve; in situ metrology.

\section{Introduction}

Perfect silicon single crystals are widely used as X-ray monochromators at third-generation synchrotron light sources. The beam footprint on the monochromator crystal (X-ray beam illuminated area) is variable and typically much smaller than the crystal size because of the variable and large Bragg angle. The beam power density on the crystal can reach several hundred $\mathrm{W} \mathrm{mm}^{-2}$. Under these circumstances, liquidnitrogen (LN2) cooling is the most effective way to limit thermal deformations. LN2-cooled silicon crystals have been widely used with great success at many synchrotron light sources (Marot et al., 1992; Rogers et al., 1995; Lee et al., 1995; Marot, 1995; also see the reviews by Bilderback et al., 2000; Mochizuki et al., 2001; Zhang et al., 2003; Chumakov et al., 2004). Numerous studies have been carried out to assess the performance limits of cryogenically cooled silicon monochromators both by finite-element analysis (FEA) modelling (Zhang, 1993; Zhang et al., 2003) and experimental testing (Lee et al., 2000, 2001; Tajiri et al., 2001; Zhang et al., 2003;

¥Present address: European XFEL, Notkestrasse 85, Hamburg 22607, Germany.
Chumakov et al., 2004). FEA simulations can determine the strain field in a heat-distorted crystal, and therefore the deformed shape of the crystal. The thermal deformation of the crystal induces rocking curve broadening, which leads to a loss of monochromatic flux. Furthermore, the shape change of the reflecting surface may deform the wavefront and thus have a negative impact on coherent and micro-focusing experiments. Additional deformations may arise, e.g. from strains introduced by the mechanical mounting of the crystal. The effective width of the rocking curve can be estimated, to a first approximation, by adding in quadrature the peak-to-peak slope error of the deformed crystal (including thermal deformation calculated by FEA and initial mechanical mounting deformation) with the theoretical intrinsic diffraction width (Zhang et al., 2003). Most studies in the literature compare the rocking curve width calculated in this way with the measured one. However, these papers present an indirect comparison between FEA results in thermal deformation and measurement results in the rocking curve broadening. The measured rocking curve broadening is balanced between two important facts. On one hand there is a change of shape of the crystal surface owing to the thermal deformations. This important 
geometrical effect (modification of the reflecting surface) is always present and it is discussed in detail in this paper. On the other hand, the thermal load produces a distortion of the diffracting crystal volume (thermal stress) that may, in cases of high thermal load, significantly modify the diffracting properties of the crystal, ranging from dynamic diffraction (no distortion of small distortions) to kinematical diffraction. For small thermal stress of the crystal (LN2-cooled silicon crystal with moderate heat load or water-cooled crystal with very low heat load), the geometrical approach is sufficient (Zhang et al., 2001, 2003; Mocella et al., 2001; Hoszowska et al., 2001). However, high heat loads produce a significant distortion of the crystal lattice, and the diffraction profile differs substantially from that of the undistorted crystal. In order to match FEA predictions with experimental rocking curves, it is necessary to solve the Takagi-Taupin equations (Takagi, 1962, 1969; Taupin, 1964, 1967) using the strain distribution in the crystal calculated by FEA. This approach has been successfully applied to water- or liquid-nitrogen-cooled monochromators (Zhang et al., 2001; Mocella et al., 2001, 2003; Hoszowska et al., 2001) where a good agreement was reported between calculated and measured rocking curves.

In order to preserve the characteristics of the photon beam in terms of flux and divergence, the design of the monochromator and cooling system has to be optimized so that the thermal slope error of the monochromator is much smaller than the angular width of the reflectivity curve (Darwin width). The ongoing European Synchrotron Radiation Facility (ESRF) Upgrade Programme (2009-2015) includes the design and construction of new beamlines [UPBLs (upgrade beamlines)], and the refurbishment and upgrade of existing beamlines. The beamline performances will be significantly improved and nano-focused beams will be routinely used owing to advances in undulator sources and beamline optics technologies. More optical elements in addition to the monochromator will be used in beamlines both upstream (white-beam mirrors) and downstream (Kirkpatrick-Baez mirrors, transfocators combining several compound refractive lenses etc.) from monochromators. Changes in the beam divergence induced by thermally deformed monochromator crystals must be considered globally as they affect the performance of the other optical elements. The overall beamline performance depends not only on the thermal slope error related to the rocking curve broadening for the photon flux preservation, but also on the thermally deformed crystal shape for the beam collimation, focusing and preservation of coherence. Therefore, in the design and optimization of the beamline optics it is essential to have accurate and reliable predictions of the shape of the optical elements under heat load. The information gained until now from the comparison between rocking curve measurements and thermal deformation calculations by FEA is insufficient to define to the specifications required for high-performance beamline design and optimization. The method presented here allows the direct experimental determination of the crystal's local thermal deformation and thus provides a more stringent test for FEA simulations.
As an example, the ESRF UPBL6 [Inelastic X-ray Scattering (IXS)] beamline requires an excellent collimation upstream of a high-resolution monochromator. The heat load on the pre-monochromator has to be considered together with the performance of a white-beam collimating mirror, as these two optical elements absorb almost all of the beam power. To keep the collimation to low values, the shape of both the mirror and pre-monochromator under heat load need to be simulated reliably and accurately. In general, the performances of the optics downstream from a crystal monochromator depend on the characteristics of the beam provided by the monochromator, that are in turn defined by its thermally deformed shape.

In order to provide a direct comparison between the FEA and experiments on the thermal deformation of the Si crystals, we have performed simultaneous heat load experiments on LN2-cooled Si crystal monochromators on several ESRF beamlines under various heat load conditions. This paper presents the experimental set-up and measurement results on the thermal slope error profile, reviews the FEA simulation of the LN2-cooled Si crystal, discusses the particularities of the FEA inputs used at the ESRF, and compares the FEA simulations with the experimental results.

\section{Experimental}

\subsection{Beamline set-up}

Heat load experiments on LN2-cooled Si monochromator crystals at the ESRF have been conducted simultaneously at three ESRF beamlines: ID06 (Techniques and Instrumentation Test beamline), ID18 (Nuclear Resonance beamline) and ID26 (X-ray Absorption and Emission Spectroscopy beamline). To vary the beam power without changing the other characteristics of the X-ray beam, the electron beam current in the storage ring was ramped from 0 to $300 \mathrm{~mA}$ in steps of $50 \mathrm{~mA}$, passing through $200 \mathrm{~mA}$ (presently the most common operation current). Two dedicated experimental sessions were allocated to these heat load tests. Two beamlines (ID06, ID18) are equipped with a $0.3 \mathrm{~mm}$ diamond window while ID26 is windowless. All beamlines have water-cooled primary slits and a LN2-cooled double silicon crystal monochromator. At the ID26 beamline there is also a water-cooled white-beam mirror upstream of the double-crystal monochromator (DCM). The white-beam mirror reduces the heat load on the DCM, but could affect the beam divergence. Therefore, it is more appropriate to concentrate on the DCMs exposed to white beam in order to assess the performance of the LN2-cooled silicon crystal monochromator. For the quantitative results presented here we use data from beamlines ID06 and ID18, though the measurements carried out at ID26 are in good qualitative agreement with the other results.

The schematic experimental set-up at beamlines ID06 and ID18 is shown in Fig. 1. There are two undulators at beamline ID06: an in-vacuum undulator U18 and a conventional undulator U32. The in-vacuum undulator U18 was set to a $8.3 \mathrm{~mm}$ gap in order to have the fundamental energy at $e_{1}=$ 


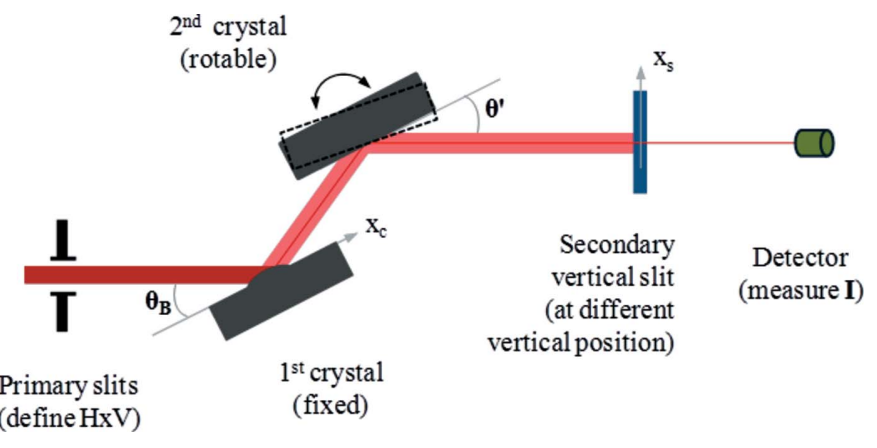

Figure 1

Set-up scheme for the thermal deformation profile measurements using rocking angle scans at various vertical positions of a narrow-gap exit slit. The rocking angle $\theta^{\prime}$ corresponding to the peak intensity in the rocking curve for the distorted first crystal is slightly different from the Bragg angle $\theta_{\mathrm{B}}$ (peak position for the undistorted crystal). The distortion of the crystal in terms of angle is given by $\Delta \theta=\theta^{\prime}-\theta_{\mathrm{B}}$. Each slit position $x_{\mathrm{s}}\left(x_{\mathrm{s}}=\right.$ 0 for the centre of the beam) allows the rocking curve to be recorded relative to the X-ray beam impinging on the first crystal surface at the position $x_{\mathrm{c}}=x_{\mathrm{s}} / \sin \theta_{\mathrm{B}}$. The thermal slope distribution $\Delta \theta\left(x_{\mathrm{c}}\right)$ of the crystal can be measured by varying the vertical positions of a narrow-gap slit after the monochromator.

$13.848 \mathrm{keV}$, and the undulator U32 to a $13.55 \mathrm{~mm}$ gap in order to have the third harmonics at the same photon energy. In addition to the $0.3 \mathrm{~mm}$-thick diamond window separating the storage ring and the beamline, there are two beryllium windows with a total thickness of $1 \mathrm{~mm}$ at beamline ID06 upstream of the monochromator. The experimental set-up at beamline ID18 is quite similar. Three U20 undulators are used with gaps set to obtain the fundamental photon energy at $e_{1}=$ $14.413 \mathrm{keV}$. The characteristics of the undulators, the beamline settings and the calculated power for a primary slits opening of $2 \mathrm{~mm} \times 1 \mathrm{~mm}(\mathrm{H} \times \mathrm{V})$ at $200 \mathrm{~mA}$ electron beam current are summarized in Table 1 . The primary slits, upstream of the monochromator, define the beam size on the monochromator and have a fixed opening [mostly $2 \mathrm{~mm} \times 1 \mathrm{~mm}(\mathrm{H} \times \mathrm{V})$ ] to maintain a constant heat load profile during the experiments. The secondary slits, after the monochromator, are set to a horizontal narrow gap $(50 \mu \mathrm{m})$, and moved vertically to isolate rocking curve data to a specific striped area on the surface of the first crystal.

The DCMs used for these tests allow the second crystal to be scanned while keeping the first one fixed. As the first crystal absorbs almost all of the incident power, the heat load on the second crystal is insignificant for the thermal deformation. Therefore the second crystal is supposed to be non-deformed with an ideal flat shape. The increase of the rocking curve width (measured after the secondary slits) for a distorted first crystal with respect to the undistorted flat crystal provides an estimate of the global slope error (peak-to-peak slope error). In order to measure the local slope error distribution on the first crystal surface, a series of rocking curves were measured at different vertical positions of the narrow-gap slit after the monochromator. Each slit position $x_{\mathrm{s}}\left(x_{\mathrm{s}}=0\right.$ for the centre of the beam) allows the recording of the rocking curve related to the photons impinging on the first crystal surface at the position $x_{\mathrm{c}}=x_{\mathrm{s}} / \sin \theta^{\prime}$, where $\theta^{\prime}=\theta_{\mathrm{B}}+\Delta \theta, \theta_{\mathrm{B}}$ is the Bragg angle
Table 1

Beamline parameters used in the experiments and some heat load calculation results at $200 \mathrm{~mA}$ electron beam current for slits opening of $2 \mathrm{~mm} \times 1 \mathrm{~mm}(\mathrm{H} \times \mathrm{V})$.

$d_{\text {src/primary slits }}$ and $d_{\text {src/mono }}$ are, respectively, the distance of the primary slits and the monochromator from the undulator source, $P_{\text {total }}$ and $P a_{\max }$ are the total power and the maximum power density of the beam from the undulator source at the position of the primary slits, $P_{\text {total-afterBeD }}$ and $P a_{\text {max-afterBeD }}\left[=P_{\mathrm{a} 0}\right.$ in equation (7)] are the total power and the maximum power density after windows, filters and just after primary slits. The beam footprint is the projected beam size on the monochromator crystal surface.

\begin{tabular}{|c|c|c|c|c|}
\hline Beamline & ID06 & ID06 & ID06 & ID18 \\
\hline Undulator & U18 & U32 & $\mathrm{U} 18+\mathrm{U} 32$ & $3 \times \mathrm{U} 20$ \\
\hline Period (mm) & 18 & 32 & & 20 \\
\hline Length (m) & 2.0 & 1.6 & & 4.8 \\
\hline Gap (mm) & 8.30 & 13.55 & & 11 \\
\hline Deflection parameter $K$ & 0.878 & 1.636 & & 0.63 \\
\hline Fund energy $(\mathrm{keV})$ & 13.848 & 4.616 & & 14.413 \\
\hline$d_{\text {src/primary slits }}(\mathrm{m})$ & 27.8 & 27.8 & 27.8 & 27 \\
\hline$H_{\text {primary slit }}(\mathrm{mm})$ & 2.0 & 2.0 & 2.0 & 2.0 \\
\hline$V_{\text {primary slit }}(\mathrm{mm})$ & 1.0 & 1.0 & 1.0 & 1.0 \\
\hline Window/filters & \multicolumn{3}{|c|}{$0.3 \mathrm{~mm}$ diamond $+1 \mathrm{~mm} \mathrm{Be}$} & $0.3 \mathrm{~mm}$ diamond \\
\hline$P_{\text {total }}(\mathrm{W})$ & 352 & 188 & 540 & 438.3 \\
\hline$P a_{\max }\left(\mathrm{W} \mathrm{mm}^{-2}\right)$ & 193.0 & 98.0 & 291 & 256 \\
\hline$\sigma_{x}(\mathrm{~mm})$ & 1.64 & 3.66 & 1.93 & 1.16 \\
\hline$\sigma_{z}(\mathrm{~mm})$ & 1.15 & 1.22 & 1.17 & 1.07 \\
\hline$P a_{\text {max-afterBeD }}\left(\mathrm{W} \mathrm{mm}^{-2}\right)$ & 170.9 & 71.0 & 241 & 236 \\
\hline$P_{\text {total-afterBeD }}(\mathrm{W})$ & 311.4 & 136.3 & 448 & 406 \\
\hline$d_{\text {src/mono }}(\mathrm{m})$ & & & 35.8 & 30 \\
\hline Bragg angle $\left({ }^{\circ}\right)$ & 25.4 & 25.4 & 25.4 & 7.9 \\
\hline Footprint $(\mathrm{H} \times \mathrm{V})(\mathrm{mm})$ & & & $2.58 \times 3.01$ & $2.22 \times 8.08$ \\
\hline
\end{tabular}

and $\Delta \theta$ the distortion of the crystal in terms of angles (see Fig. 1). These angles can be determined from the rocking curve measurements: $\theta^{\prime}$, the rocking angle at which the rocking curve reaches maximum for a distorted crystal; $\theta_{\mathrm{B}}$, the peak position for the undistorted flat crystal. For a LN2-cooled silicon crystal, the thermal slope error $\Delta \theta$ is of the order of $10 \mu \mathrm{rad}$ which is much smaller than the Bragg angle $\theta_{\mathrm{B}}\left(25.4^{\circ}\right.$ for the ID06 monochromator). The exit beam after the monochromator is parallel to the incident beam owing to the double crystals. The thermal deformation of the first crystal can induce a parallel beam displacement $\delta x_{\mathrm{s}}$ at the position of the scanning slit. This displacement can be calculated as $\delta x_{\mathrm{s}}=$ $L \Delta \theta \tan \left(\theta_{\mathrm{B}}\right)$, where $L$ is the distance between the exit beam and incident beam, and approximately equal to $15 \mathrm{~mm}$. For $\Delta \theta=10 \mu \mathrm{rad}$ of thermal slope error, the beam position shift $\delta x_{\mathrm{s}} \simeq 0.071 \mu \mathrm{m}$ is much smaller than the scanning slit gap $(50 \mu \mathrm{m})$, and independent of the distance between the scanning slits and the monochromator. An advantage of this method is to only use the peak positions of the different rocking curves. Therefore it is independent of the diffraction profile so it also works for the case of large thermal deformation where diffraction profiles are usually calculated by Takagi-Taupin equations. By varying the vertical position of the narrow-gap slit, we obtain the angular distribution $\Delta \theta\left(x_{\mathrm{c}}\right)$, also called the thermal slope error distribution. The integral of this angular distribution gives the deformation profile; the derivative of this angular distribution gives the curvature (approximately the inverse of the radius) distribution. In summary, the traditional rocking curve width measurement with full beam gives only the rocking curve broadening, 
providing indirect and global information on the thermal deformation, while measurement of the rocking curve peak angular position through a vertical narrow-gap slit at different slit positions enables the slope profile of the deformed crystal to be recorded, thereby providing direct and local information on the thermal deformation.

\subsection{Calorimetry description}

The thermal and mechanical properties of silicon versus temperature are strongly non-linear in the $\mathrm{LN} 2$ temperature range. The temperature and thermal deformation of the LN2cooled Si crystal are very sensitive to the heat load. Therefore, for FEA simulations it is essential to use very accurate values of the power from the beam used in the experiment. To fulfil this requirement, a calorimeter was developed at the ESRF to measure the X-ray beam power (Zhang \& Biasci, 2005). It consists of a $100 \mathrm{~mm} \times 50 \mathrm{~mm} \times 50 \mathrm{~mm}$ copper block with a hole of $10 \mathrm{~mm}$ in diameter and $70 \mathrm{~mm}$ deep, through which the $\mathrm{X}$-ray beam is sent. In this geometry the power losses by scattering outside the copper block are negligible. The calorimeter is mounted in a vacuum vessel, supported by a thermal insulator and was not cooled during the measurement. A Pt-100 temperature sensor attached to the cooper block is used to measure the temperature response of the calorimeter. Initially, the temperature $T_{0}$ of the copper block is uniform. The X-ray beam was sent into the hole for approximately $\Delta t=$ $60 \mathrm{~s}$ and then switched off by a fast shutter. The temperature measured at the position of the Pt-100 sensor stabilizes at a constant value $T_{\mathrm{m}}$ about $120 \mathrm{~s}$ after the beam has been switched off. During this short period the energy loss is less than $0.6 \%$ of the total incident beam energy. Therefore, the uniform temperature $T_{\mathrm{m}}$ is practically the average temperature of the calorimeter at the moment the beam is switched off. The average beam power $P$ can be calculated from the temperature increase during this period $\Delta T=T_{\mathrm{m}}-T_{0}$ as

$$
P=m / \Delta T \int_{T_{0}}^{T_{\mathrm{m}}} c_{\mathrm{p}} \mathrm{d} T=m c_{\mathrm{p}} \Delta T / \Delta t
$$

where $m$ is the mass of the copper block and $c_{\mathrm{p}}$ is the heat capacity of copper. A single power measurement takes about $180 \mathrm{~s}$ using this method. Taking into account the temperature measurement accuracy and the power loss, the accuracy of the calorimeter is estimated to be in the range 0 to $-1.2 \%$.

\subsection{Results}

2.3.1. Beam power and calorimetry. In order to calculate the thermal deformations of the monochromator crystals, the power distribution of the incident white beam must be known. The photon flux and power produced by storage rings, including the undulator emission, can be calculated precisely using classical electrodynamics. Many codes are used in synchrotron light sources, such as SRW (Chubar, 1998), Spectra (Tanaka \& Kitamura, 2000) and XOP (Sánchez del Río \& Dejus, 2011). For the most common cases they produce similar results. We use $S R W$ to compute the undulator power distribution in the plane normal to the beam at the position of
Table 2

Beam power measured by a calorimeter $\left(P_{\text {meas }}\right)$ compared with power calculated using the $S R W$ code $\left(P_{\mathrm{SRW}}\right)$, different electron beam currents $(I)$ and primary slits horizontal $(\mathrm{H})$ and vertical $(\mathrm{V})$ apertures.

The power absorption by windows and filters is considered in the calculated power.

\begin{tabular}{lllllll}
\hline Date & Undulator & $\begin{array}{l}\mathrm{H} \times \mathrm{V} \\
(\mathrm{mm})\end{array}$ & $\begin{array}{l}I \\
(\mathrm{~mA})\end{array}$ & $\begin{array}{l}P_{\text {meas }} \\
(\mathrm{W})\end{array}$ & $\begin{array}{l}P_{\text {SRW }} \\
(\mathrm{W})\end{array}$ & Difference \\
\hline \multirow{2}{*}{2004} & $\mathrm{U} 42 \mathrm{~g} 12.1$ & $3 \times 3$ & 60 & 163 & 181 & $-10 \%$ \\
& & & 85 & 224 & 256 & $-12 \%$ \\
\multirow{2}{*}{2010} & \multirow{2}{*}{$\mathrm{U} 18 \mathrm{~g} 8.3+\mathrm{U} 32 \mathrm{~g} 13.55$} & $2 \times 1$ & 100 & 201 & 224 & $-10 \%$ \\
& & & 200 & 401 & 448 & $-10 \%$ \\
& & & 240 & 475 & 538 & $-12 \%$ \\
\hline
\end{tabular}

the primary slits. Then we make Gaussian fits, and deduce three parameters to describe the power distribution: $P_{\mathrm{a} 0}$, peak power density; and $\sigma_{\mathrm{H}}$ and $\sigma_{\mathrm{V}}$, the horizontal and vertical RMS beam sizes.

Many calorimetry experiments have been carried out to compare the experimental results with the theoretical predictions. The X-ray beam incident power to the LN2cooled silicon crystal monochromators at the ID06 beamline was first measured in 2004 when the calorimeter described in the previous section was installed, and then in 2010 when the heat load experiments on LN2-cooled silicon crystals were carried out. The power calculated by the $S R W$ code takes into account the source parameters: the attenuation by diamond and beryllium windows, the primary slits aperture and the distance from the source. Table 2 shows the beam power measured by the calorimeter and the comparison with the power calculated using the $S R W$ code. The undulators, the aperture of the primary slits and electron beam current were different between the measurements carried out in 2004 and in 2010. The measured beam power is $10-12 \%$ smaller than the calculated result. Considering the accuracy of the calorimeter, the power from the undulator in beamline ID06 is about $10 \%$ lower than the calculated power. This discrepancy may be explained, for instance, by the accuracy of the primary slits opening and positioning, the accuracy of the thickness of the windows and purity of the window material upstream of the monochromator, the accuracy of the calorimeter, and the accuracy of the undulator parameters (magnetic field, period, gap and length). This discrepancy could be different from one beamline to another, and will be the subject of further investigation. Power losses due to beam scattering on the silicon crystal were estimated to be about $4 \%$ of the incident power to the DCM at beamline ID06 according to a Monte Carlo simulation (Secco \& Sánchez del Río, 2011). From the footprint on the first crystal the observing solid angle covered by the second crystal is about 0.4 times half space (calculated from a drawing of the monochromator with the two crystals). The heat load on the second crystal is then about $1.6 \%$ of the incident power, and distributed over the surface of the second crystal which is effectively LN2-cooled. The heating and thermal deformation of the second crystal by Compton scattering is therefore negligible. Finally, the power absorbed by the first Si crystal at beamline ID06 is $f p_{\text {cor }}=14 \%$ lower than 
the calculated power $(10+4 \%=14 \%)$. This correction factor will be used to calculate the effectively absorbed power by the first crystal.

In addition, the power absorbed by the first crystal can be estimated as the power evacuated by the LN2 flow in the cooled silicon crystal by measuring the increase of the LN2 temperature $\Delta T_{f}$ between the monochromator outlet and inlet,

$$
P=Q \rho c_{\mathrm{p}} \Delta T_{f},
$$

where $\rho$ and $c_{\mathrm{p}}$ are the density and heat capacity of LN2, and $Q$ is the LN2 flow rate in volume per unit of time. In general, this approach provides a direct estimation of the absorbed power. However, it is accompanied by several sources of uncertainty. In particular, the flow rate is estimated from the measured pressure drop. As the pressure drop curve of the LN2 cooling loop versus the flow rate is quite flat over a large flow rate range, there can be a rather high degree of uncertainty of the flow rate, i.e. in the range 150 to $250 \mathrm{~L} \mathrm{~h}^{-1}$ for the measured pressure drop. This problem can be solved using the results obtained in the laboratory measurements of the temperature increase as a function of the calibrated power load. Another source of uncertainty is the small value of the temperature difference between the outlet flow and inlet flow: $2.7 \mathrm{~K}$ for the ID06 monochromator and $3 \mathrm{~K}$ for the ID18 monochromator with primary slits opening of $2 \mathrm{~mm} \times 1 \mathrm{~mm}$ $(\mathrm{H} \times \mathrm{V})$ and at $200 \mathrm{~mA}$. Thus, the method based on the measurement of the LN2 temperature difference between the outlet and the inlet of the monochromator is used only for approximate power estimation with an uncertainty of about $20 \%$.

2.3.2. Rocking curve width. Rocking curves at a Bragg angle of $25.4^{\circ}$ were measured using the ID06 beamline monochromator. The $\mathrm{Si}(333)$ reflection was selected, because the intrinsic rocking curve width of the double-crystal monochromator at this reflection $\left(e_{3}=13.848 \mathrm{keV}\right)$ is $6.2 \mu \mathrm{rad}$ (FWHM), which is significantly smaller than that for the reflection $\mathrm{Si}(111)(96.2 \mu \mathrm{rad}$ at $4.616 \mathrm{keV})$. Thus, the $\mathrm{Si}(333)$ reflection at $13.848 \mathrm{keV}$ allows much smaller crystal distortions to be observed.

Rocking curves were measured for different positions of a vertically translating narrow-gap slit downstream from the monochromator, as explained in $\$ 2.1$, and shown in Fig. 2. The FWHM of these rocking curves is approximately constant $(\sim 9.5 \mu \mathrm{rad})$ at an electron beam current $I=101 \mathrm{~mA}$ and a power absorbed by the crystal $P=194.5 \mathrm{~W}$. The thermal slope error including the initial deformation of the crystal is estimated to be $7.2 \mu \mathrm{rad}$ FWHM (or $3.1 \mu \mathrm{rad}$ RMS assuming Gaussian distributions) by de-convolving the experimental FWHM with the intrinsic (i.e. for an undistorted first crystal) Darwin curve broadening $\theta_{\text {intr }}=6.2 \mu \mathrm{rad}(\mathrm{FWHM})$. The angular shift of the peak of each rocking curve results directly in the slope of the first crystal at each $x_{\mathrm{c}}$ coordinate. It is obtained from the peak positions of the Gaussian profiles fitted to the rocking curves. The measured slope profile of the crystal is a weighted average over the transverse footprint

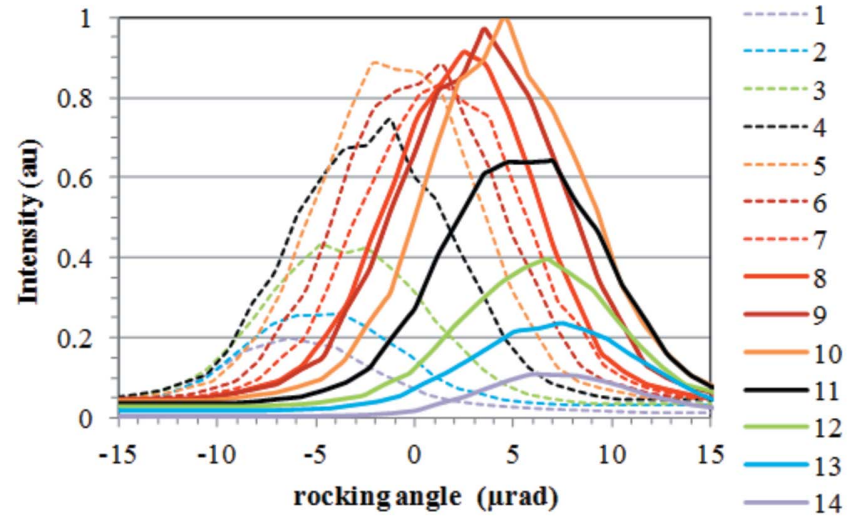

Figure 2

Rocking curves of the monochromator at beamline ID06 for the $\mathrm{Si}(333)$ reflection at $13.848 \mathrm{keV}$, an electron beam current of $101 \mathrm{~mA}$ and a power absorbed by the crystal $P=194.5 \mathrm{~W}$. The primary slits opening is $2 \mathrm{~mm} \times$ $1 \mathrm{~mm}(\mathrm{H} \times \mathrm{V})$. The vertical opening of the secondary slit is $0.05 \mathrm{~mm}$. The curves 1 and 14 correspond to the most upstream and downstream position, respectively, on the crystal surface. Each curve $(i)$ is measured at a position separated by $0.47 \mathrm{~mm}$ from the previous one $(i-1)$ along the beam direction on the crystal surface.

width, with the highest weight coefficient along the central axis.

2.3.3. Deformed crystal slope and profiles. The slope distribution along the crystal footprint length obtained as described above can be used to calculate the deformed crystal profile under the X-ray beam power by the integration of the slope distribution over the crystal coordinate $x_{\mathrm{c}}$. Results in thermal slope distribution and deformed profile from experiments carried out at beamlines ID18 and ID06 are shown in Figs. 3(a)-3(d). At small beam current (low heat load), the shape of the crystal is flat or slightly concave. As the electron beam current (power) is increased, the profile concavity increases, and then decreases to form a mostly flat surface. The electron beam current at which this approximately flat surface is obtained depends on the slit aperture and on the Bragg angle, i.e. on both the total power and the power density. When the electron beam current is increased further, the crystal becomes deformed into a convex shape, forming a bump that grows quickly with the electron beam current or heat load on the crystal.

\section{Finite-element modelling}

The FEA simulations rely on the accurate description of the problem, including the boundary conditions. Several critical issues are discussed here: the material properties of silicon, the beam power absorbed by the crystal, and the finite-element model with mechanical and thermal boundary conditions. The FEA software used is ANSYS (release 14.0, ANSYS Inc.).

\subsection{Material properties}

The temperature distribution on the crystal monochromator depends on the impinging X-ray beam power distribution, the thermal conductivity $k$ of the crystal material (silicon for the present study), and the crystal geometry and 


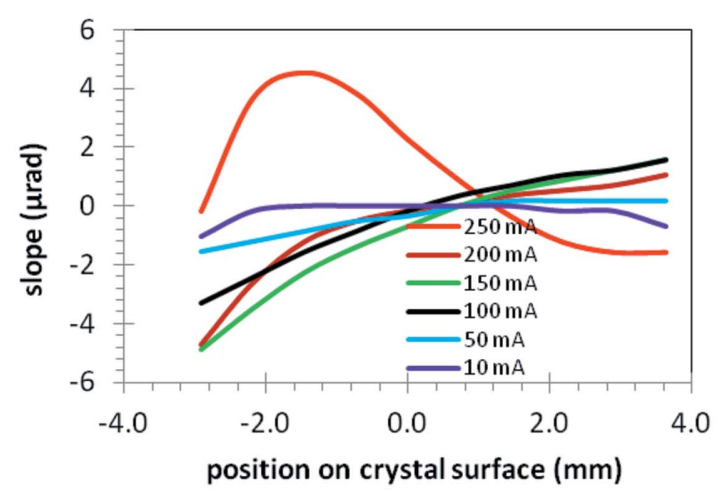

(a)

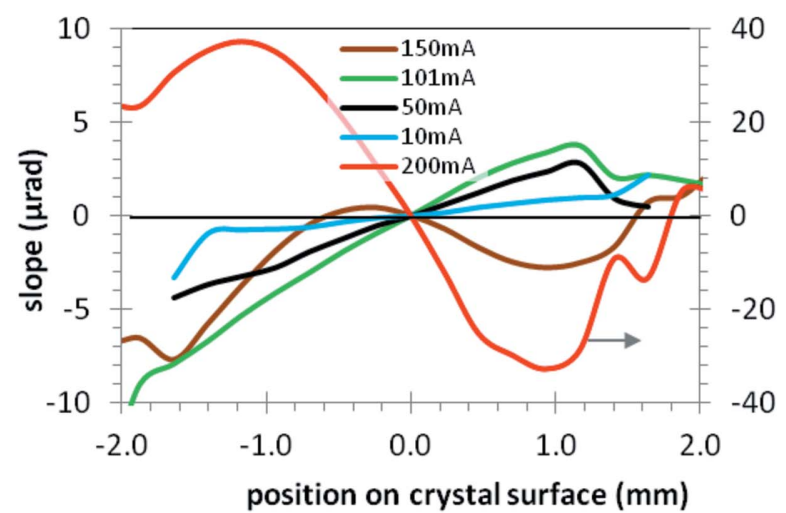

(b)

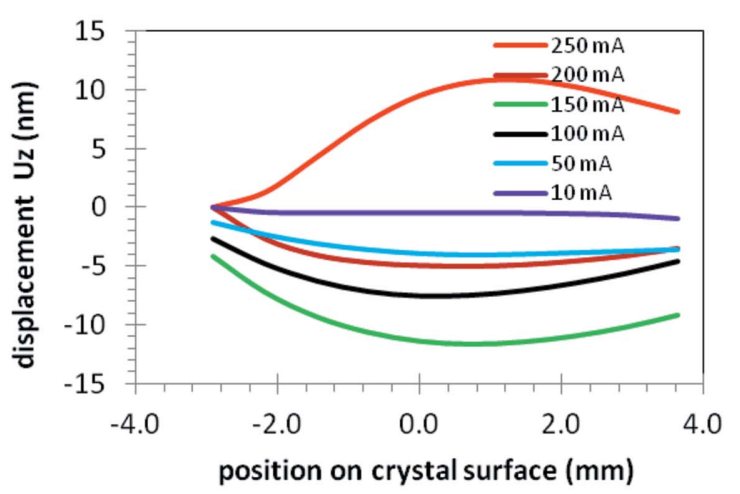

(c)

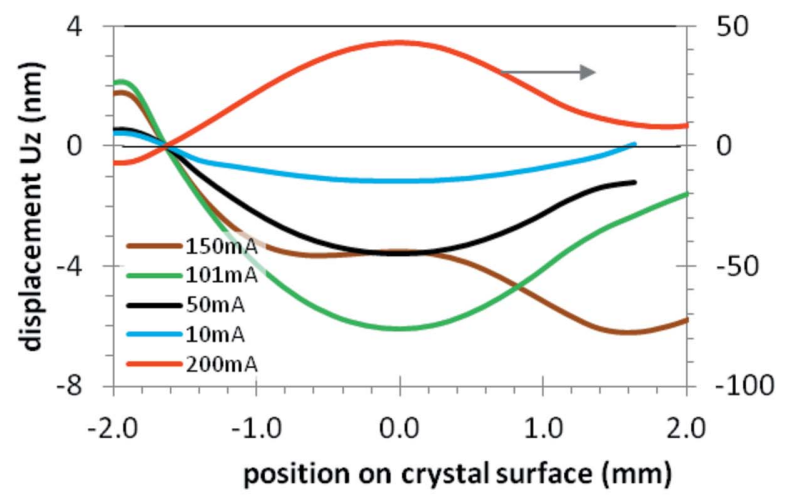

(d)

Figure 3

Thermal slope $(a, b)$ and displacement profile $(c, d)$ for the first silicon crystal versus the crystal coordinate $x_{\mathrm{c}}$ at various electron beam currents (power load) at the two beamlines ID18 $(a, c)$ and ID06 $(b, d)$. The right-hand scale in $(b, d)$ is for the case of $200 \mathrm{~mA}$ electron beam current (red line).

cooling conditions. The temperature gradient induces thermal deformation of the crystal. The related material property is the thermal expansion coefficient $\alpha$. For materials with constant coefficients $k$ and $\alpha$, the thermal deformation is inversely proportional to the thermal conductivity $k$ and proportional to the thermal expansion coefficient $\alpha$. The ratio $\alpha / k$ is often used to estimate the thermal deformation. For silicon, these two material properties are strongly temperature-dependent (Fig. 4a). The ratio $\alpha / k$ of silicon at $\mathrm{LN} 2$ temperature $(77 \mathrm{~K}$ at $1 \mathrm{~atm}$ ) is much lower than at room temperature. Therefore, LN2 cooling can significantly reduce the thermal deformation of the silicon crystal compared with water cooling.

The thermal strain of a solid body when the temperature varies from $T_{\text {ref }}$ to $T$ is

$$
\varepsilon=\int_{T_{\text {ref }}}^{T} \alpha(T) \mathrm{d} T
$$

For silicon, the thermal expansion coefficient $\alpha$ is zero at $125 \mathrm{~K}$, but this integral (thermal strain) is not zero when the temperature of the silicon crystal varies from the LN2 temperature (for example, $T_{\text {ref }}=77 \mathrm{~K}$ ) to $125 \mathrm{~K}$. When $\alpha$ is constant, this expression becomes

$$
\varepsilon=\alpha\left(T-T_{\text {ref }}\right) .
$$

For temperature-dependent $\alpha$, the strain can still be calculated using a formula similar to (4), but replacing $\alpha$ with the secant coefficient of thermal expansion, $\alpha^{\text {se }}$ (see $\$ 2.1 .3$ of the ANSYS documentation Theory Reference),

$$
\varepsilon=\alpha^{\mathrm{se}}\left(T-T_{\text {ref }}\right)
$$

where

$$
\alpha^{\mathrm{se}}=\left[\int_{T_{\mathrm{ref}}}^{T} \alpha(T) \mathrm{d} T\right] /\left(T-T_{\mathrm{ref}}\right)
$$

Note that $\alpha^{\text {se }}$ depends on both the reference temperature $T_{\text {ref }}$ and the temperature $T$. The data for the thermal expansion coefficient given in the literature are usually the values of $\alpha$ as a function of temperature, as in Touloukian et al. (1970b). This is the so-called 'instantaneous' $\alpha$ in the ANSYS documentation. The secant coefficient of the thermal expansion $\alpha^{\text {se }}$ calculated by (6) at $T_{\text {ref }}=77 \mathrm{~K}$, and the thermal strain $\varepsilon_{\text {th }}$ by (3) for silicon are shown as a function of temperature in Fig. 4(b). It is noticeable that the instantaneous coefficient of the thermal expansion $\alpha$ is zero at $T=125 \mathrm{~K}$, but the secant coefficient of thermal expansion and the thermal strain are zero at $T=165 \mathrm{~K}$. Let us consider a stress-free silicon block cooled down to the LN2 temperature $T_{\text {ref }}=77 \mathrm{~K}$, then uniformly warmed up. From temperature $T_{\text {ref }}=77 \mathrm{~K}$ to $125 \mathrm{~K}$, it is in thermal contraction down to $\varepsilon_{\mathrm{th}}=-16.5 \times 10^{-6}$ at $125 \mathrm{~K}$ [equation (3) or Fig. 4(b) curve $\varepsilon_{\mathrm{th}}$ ]. Above $125 \mathrm{~K}$, the silicon crystal is in thermal expansion that compensates the accumulated thermal contraction from $77 \mathrm{~K}$ to $125 \mathrm{~K}$, and 


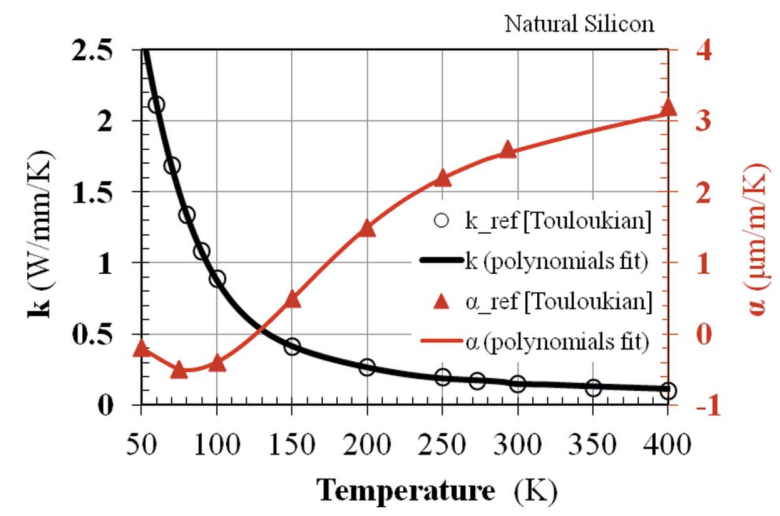

(a)

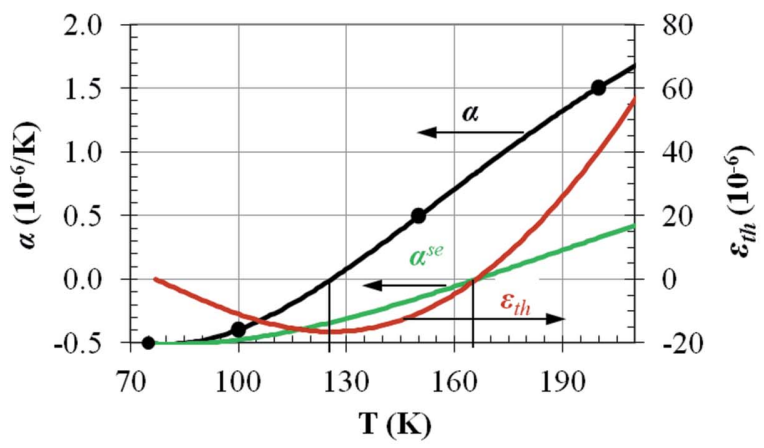

(b)

Figure 4

(a) Thermal conductivity and thermal expansion coefficient of silicon versus temperature. The data represented by circles and triangles are from Touloukian et al. (1970a); the continuous lines are polynomials fits. (b) Thermal expansion coefficients: $\alpha$ (black line), secant $\alpha^{\text {se }}$ (green line) and thermal strain $\varepsilon_{\text {th }}$ (red line) versus temperature for a silicon crystal, the reference temperature being $T_{\text {ref }}=77 \mathrm{~K}$.

then goes back to its initial size (no deformation) at $165 \mathrm{~K}$. For a LN2-cooled silicon crystal under X-ray power, the temperature of the crystal is increased from the initial LN2 temperature to a higher but not uniform temperature. The maximum temperature of the crystal is located in the region that is illuminated by the X-ray beam. When this maximum temperature is near $T=165 \mathrm{~K}$, the thermal deformation of the crystal reaches a local minimum (but not zero deformation since the temperature in the crystal is not uniform). At $T=$ $125 \mathrm{~K}$, the thermal deformation of the silicon crystal is not minimum (see Fig. $4 b$ ) but mostly thermally contracted. The idea spread throughout the synchrotron radiation community that the LN2-cooled silicon crystal has zero thermal deformation at $125 \mathrm{~K}$ is incorrect. (See Appendix $A$ for a discussion on the ANSYS command for the temperature-dependent thermal expansion coefficient.)

The anisotropic mechanical properties of silicon (Wortman \& Evans, 1965; Zhang, 2009), using the stiffness coefficient matrix for $\mathrm{Si}(111)$, have been used in the FEA of this study.

\subsection{Beam power absorbed by the crystal}

The thermal deformation of the monochromator crystal under heat load depends not only on the material properties as discussed above but also on the beam parameters related to the beam power distribution that are discussed here.

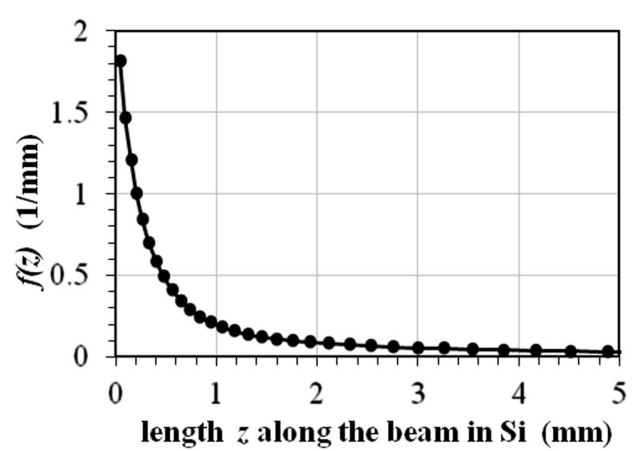

Figure 5

Volume power absorption function $f(z)$ along the beam path in silicon. The undulator source $\mathrm{U} 18+\mathrm{U} 32$, a $0.3 \mathrm{~mm}$ diamond and a $1 \mathrm{~mm}$-thick beryllium window have been considered in the calculation. This function $f(z)$ is an attenuation function for silicon averaged over all of the relevant photon energy spectrum up to $100 \mathrm{keV}$.

For an undulator beam the spatial distribution of the power in a plane normal to the beam at a distance $d$ from the source is well approximated by a Gaussian distribution with parameters $\sigma_{\mathrm{V}}$ and $\sigma_{\mathrm{H}}$. In the case of beamline ID06 at the crystal position $d=35.8 \mathrm{~m}$ from the undulator source, we have $\sigma_{\mathrm{V}}=$ $1.55 \mathrm{~mm}$ and $\sigma_{\mathrm{H}}=3.28 \mathrm{~mm}$. Heat load experiments with LN2cooled silicon crystals have been performed at different electron beam currents. The power $P_{\mathrm{v}}$ absorbed by a unit volume of the Si crystal at electron beam current $I$ is given by

$$
\begin{aligned}
P_{\mathrm{v}}(x, y, z)= & P_{\mathrm{a} 0}\left(1-f p_{\text {cor }}\right)\left(I / I_{\text {ref }}\right) f(z) \\
& \times \exp \left[\left(-x^{2} / \sigma_{\mathrm{H}}^{2}\right)-\left(y^{2} / \sigma_{\mathrm{V}}^{2}\right)\right],
\end{aligned}
$$

where $P_{\mathrm{a} 0}\left(\right.$ in $\mathrm{W} \mathrm{mm}{ }^{-2}$ ) is the calculated power density at the position of the crystal, $f p_{\text {cor }}=0.14$ is a correction factor for the difference between the calculated power and absorbed power (see §2.3.1), $I_{\text {ref }}$ is the nominal electron beam current (200 mA), $f(z)$ is the power absorption function of silicon calculated as the ratio of the absorbed power $P_{\text {abs-by- } \Delta z \mathrm{Si}}$ by a slice of silicon (thickness $\Delta z$ ) and incident power $P_{\text {inc }}$ as $f(z)=$ $P_{\text {abs-by- } \Delta z \mathrm{Si}} / P_{\text {inc }} / \Delta z$. The function $f(z)$ is an attenuation function averaged over the entire photon energy spectrum up to $100 \mathrm{keV}$. Results of $f(z)$ are plotted in Fig. 5. The coordinate system here is $O-x y z$, where $x$ and $y$ are the horizontal and vertical axes, respectively; the $z$-axis is along the beam path, and the origin of the coordinate system is point $O$ at the centre of the footprint on the crystal surface. In a coordinate system lying on the crystal surface $O-X Y Z$, a projection factor $\sin \theta_{\mathrm{B}}$ has to be applied accordingly. The units of the different terms are: $P_{\mathrm{v}}, \mathrm{W} \mathrm{mm}{ }^{-3} ; P_{\mathrm{a} 0}, \mathrm{~W} \mathrm{mm^{-2 }} ; I, \mathrm{~mA} ; x, y, \sigma_{\mathrm{H}}, \sigma_{\mathrm{V}}, \mathrm{mm}$; $f(z), \mathrm{mm}^{-1}$.

\subsection{Finite-element model}

The first silicon crystal of the ID06 monochromator (as well as those of ID26 and ID18) is cooled from the two sides by copper blocks with a $0.5 \mathrm{~mm}$-thick foil of indium on each of the two interfaces (Fig. 6a). The indium foils ensure a good thermal contact between the copper blocks and the silicon crystal. A small pressure maintains them in contact without 


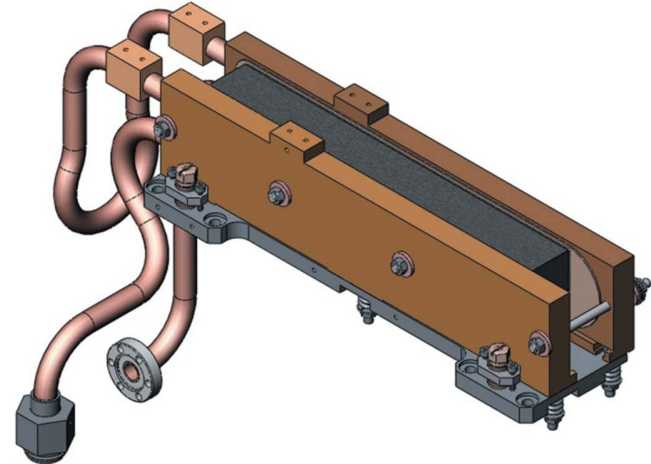

(a)

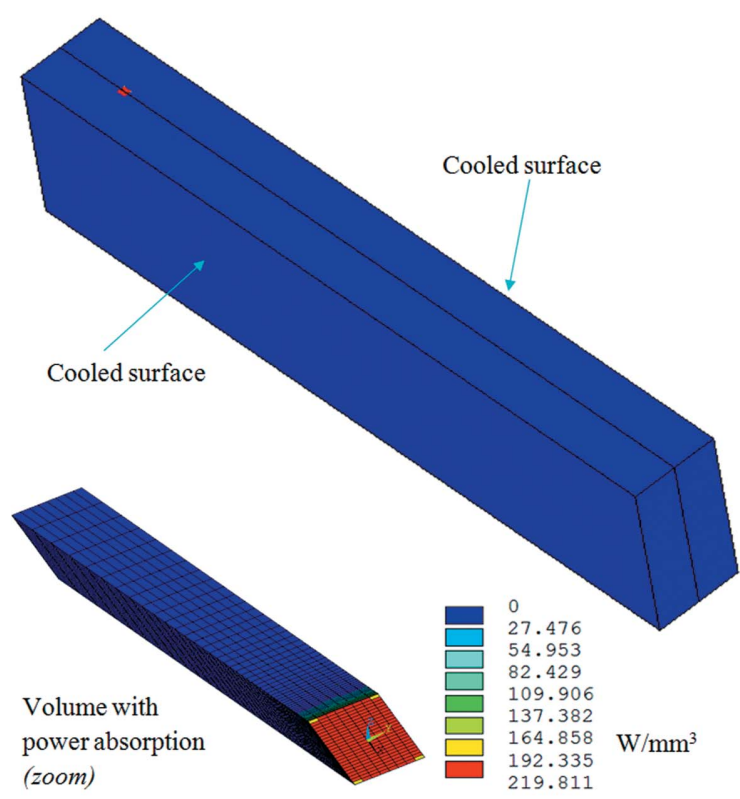

(b)

\section{Figure 6}

Finite-element model for the ID06 monochromator: $(a)$ first silicon crystal with LN2 cooling system, (b) finite-element model of the first silicon crystal. The scale for the power density corresponds to a total absorbed power of $385.2 \mathrm{~W}$ under the following conditions: primary slits opening $=2 \mathrm{~mm} \times 1 \mathrm{~mm}(\mathrm{H} \times \mathrm{V})$; beam current $I=200 \mathrm{~mA}$; power correction factor $f p_{\text {cor }}=14 \%$.

deforming the crystal. In this study the finite-element modelling is applied only to the silicon crystal, a block $240 \mathrm{~mm}$ long, $50 \mathrm{~mm}$ thick and $28 \mathrm{~mm}$ wide (Fig. $6 \mathrm{~b}$ ), as in previous studies (Zhang, 1993; Zhang et al., 2001, 2003). An effective convection cooling coefficient $h_{\mathrm{cv}}$ is applied to the side surfaces of the crystal in contact with the copper blocks. This cooling coefficient depends on the LN2 flow rate in the cooling blocks and the thermal contact resistance, and is determined from the measured temperature of the crystal. The absorbed power by the volume of the silicon crystal (shown in Fig. 6b) is calculated by equation (7) using the function $f(z)$ shown in Fig. 5. For the slits opening $2 \mathrm{~mm} \times 1 \mathrm{~mm}(\mathrm{H} \times \mathrm{V})$, an electron beam current of $I=200 \mathrm{~mA}$ and a power correction factor of $f p_{\text {cor }}=$ 0.15 , the total absorbed power is $385.2 \mathrm{~W}$. At the Bragg angle of $\theta_{\mathrm{B}}=25.4^{\circ}$, the footprint is close to one end of the crystal as shown in Fig. 6(b), the small red point on the left part of the crystal surface. ${ }^{\mathbf{1}}$ The temperature of the crystal is measured at a point on the left end of the crystal close to the bottom, well below the power absorption volume.

The X-ray beam was assumed to impinge along the centre of the crystal, symmetrically on the crystal in the sagittal direction. Therefore, only half of the crystal needs to be modelled. The symmetrical boundary conditions were applied to the central plane parallel to the cooling surfaces. As our interest is in calculating the thermal deformation, it was assumed that the crystal is free of mechanical constraints related to the copper blocks.

The volume of the crystal absorbing the power is an inclined rectangular prism $20 \mathrm{~mm}$ in length with the beam footprint $(2.58 \mathrm{~mm} \times 3.01 \mathrm{~mm})$ as base (Fig. $6 b)$. The footprint is firstly meshed in rectangular two-dimensional elements, and then extruded to three-dimensional elements with progressively increasing element size along the beam path ( $z$-axis) over a total length $L_{z}=20 \mathrm{~mm}$. This corresponds to a silicon thickness (along the $Z$-axis, normal to the crystal surface) of $t_{Z}=$ $20 \sin \theta_{\mathrm{B}}=8.58 \mathrm{~mm}$. The height of the variable element mesh $\Delta Z_{i}$ is related to the variable thickness $\Delta z_{i}$ used for the calculation of the volume power absorption function as: $\Delta Z_{i}=$ $\Delta z_{i} \sin \theta_{\mathrm{B}}$. After a total length of $L_{z}=20 \mathrm{~mm}$ of silicon, the transmitted power (up to $100 \mathrm{keV}$ high-energy photons) is only $3.3 \%$ of the incident power. The crystal thickness is $50 \mathrm{~mm}$, which is equivalent to a $116.6 \mathrm{~mm}$ length along the X-ray beam path with a Bragg angle of $25.4^{\circ}$. The power transmitted through the whole crystal is negligible $(0.01 \%)$. In order to limit the size of the model, all the absorbed power is input into the inclined rectangular prism volume. Once this inclined prism volume is meshed, the rest of the silicon crystal is then meshed semi-automatically with several intermediate transition volumes.

\section{FEA results and comparison with the experiment}

\subsection{Temperature distribution and cooling coefficient}

The effective cooling coefficient $h_{\mathrm{cv}}$ depends mostly on the thermal contact resistance at the interface between the silicon crystal and the copper cooling block. The key influencing parameters on this thermal contact resistance are the applied pressure (contact pressure) and the surface state of the contact bodies at the interface (Vallet \& Zhang, 2003; Marion et al., 2004). Various estimations (Zhang, 1993; Zhang et al., 2003) show that this effective cooling coefficient $h_{\mathrm{cv}}$ for LN2-cooled silicon and copper blocks is mostly in the range 1000$5000 \mathrm{~W} \mathrm{~m}{ }^{-2} \mathrm{~K}^{-1}$. The exact value of the cooling coefficient $h_{\mathrm{cv}}$ will be determined when comparing experimental and FEA results.

\footnotetext{
${ }^{\mathbf{1}}$ For the ID06 monochromator the centre of rotation is located at half the height between the white and monochromatic beams. Therefore the beam footprint moves across the surface of the first crystal when the Bragg angle is changed. In this geometry it is not necessary to translate the second crystal parallel to the beam. Experiments were carried out at large Bragg angle, where the beam footprint is small and is located relatively close to the centre of rotation, i.e. the downstream edge of the crystal.
} 


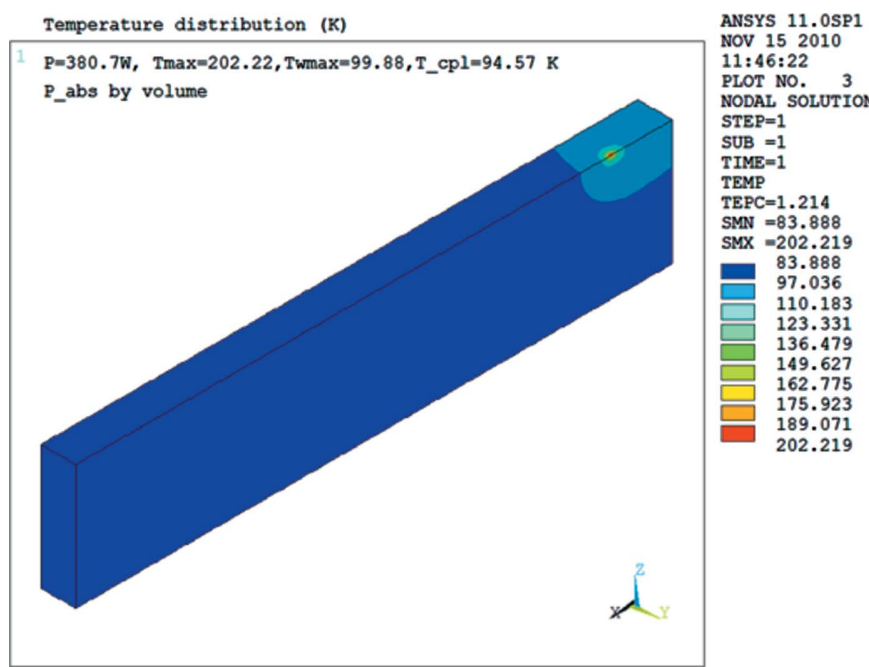

ID6 crystal $\mathrm{HxV}=2 \times 1 \quad(\mathrm{Tf}=82.35 \mathrm{~K}, \mathrm{HcV}=2872 \mathrm{~W} / \mathrm{m} 2 / \mathrm{C}), I=200 \mathrm{~mA}$

\section{Figure 7}

Temperature distribution of the first crystal of the ID06 monochromator for the primary slits opening $2 \mathrm{~mm} \times 1 \mathrm{~mm}(\mathrm{H} \times \mathrm{V})$ and electron beam current $I=200 \mathrm{~mA}(P=385.2 \mathrm{~W})$.

FEA performed by assuming a cooling coefficient $h_{\mathrm{cv}}$ calculates the temperature distribution in the silicon crystal, in particular at the position where the temperature is measured. The effective cooling coefficient $h_{\mathrm{cv}}$ can be deduced by fitting the FEA to the measurement temperature at this position. We made this fitting for one heat load case, and then used the deduced cooling coefficient in the calculations for all other heat load conditions. A final fine-tuning of the cooling coefficient $h_{\mathrm{cv}}$ was carried out to obtain the best temperature fitting. The deduced effective cooling coefficient $h_{\mathrm{cv}}$ is $2906 \mathrm{~W} \mathrm{~m}^{-2} \mathrm{~K}^{-1}$ from a first experiment carried out at beamline ID06, and $2656 \mathrm{~W} \mathrm{~m}^{-2} \mathrm{~K}^{-1}$ from a second experiment performed at the same beamline five months later. The difference between these two values is only $9 \%$ and is mostly due to the variation of the LN2 flow rate. The thermal deformation of the LN2-cooled silicon crystal is almost independent of the cooling coefficient when the absorbed power is in the linear region (Zhang et al., 2003). As an example, the temperature distribution of the silicon crystal is shown in Fig. 7 for the case of slits opening $2 \mathrm{~mm} \times 1 \mathrm{~mm}(\mathrm{H} \times \mathrm{V})$ and a beam current $I=200 \mathrm{~mA}$. The maximum temperature of the crystal in this case reaches $202 \mathrm{~K}$. The region with temperatures higher than $100 \mathrm{~K}$ is small and surrounds the beam footprint.

The calculated temperatures at the position of the thermocouple are compared with those measured for different test conditions (electron beam current, slits opening) (Fig. 8). The difference between the calculated and measured temperatures is smaller than $0.6 \mathrm{~K}$. An excellent correlation factor (0.9995) between the calculated temperature and measured temperature confirms the good choice of the thermal boundary conditions (the cooling coefficient and the power load) used in the FEA.

Similar FEA simulations compared with the experimental results at the ID18 beamline led to an effective convective

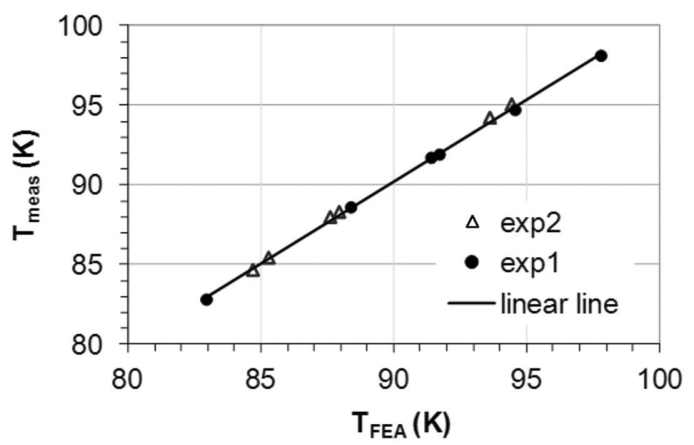

Figure 8

Calculated temperatures by FEA at the position of the thermocouple are compared with the measured ones for different test conditions for the ID06 silicon crystal. Experiment data come from two dedicated heat load test sessions (exp 1 and $\exp 2)$. The line is a guide for the eyes. The correlation factor between the calculated and measured temperatures is 0.9995 .

cooling coefficient of $h_{\mathrm{cv}}=3510 \mathrm{~W} \mathrm{~m}^{-2} \mathrm{~K}^{-1}$. The mounting of the silicon crystal and copper cooling blocks is not identical in these two beamlines. The contact pressure and the flow rate were also different. Considering these effects, the effective cooling coefficients are comparable between both beamlines ID06 and ID18.

\subsection{Thermal deformation of the silicon crystal}

The calculated temperature distribution is used to compute the pure thermal deformation in the silicon crystal. It is supposed that the crystal block is free of mechanical constraints although in practice the cooling copper block assembly could introduce mechanical stress and strain. The initial state is chosen to be the crystal shape with mechanical strain due to fabrication/mounting and the cooling down from room temperature to the LN2 temperature just before switching on the heat load. Then, the thermal deformation due to the heat load is superposed linearly to the initial state, and can be separately treated. It is possible to estimate the initial deformation of the crystal by comparing FEA results with the experimental results at a very small power load. As an example, this technique was used to estimate at 1.1 arcsec the initial slope error due to mounting, fabrication and cooling down to LN2 temperature of the channel-cut monochromator crystal at the ESRF beamline ID09 (Zhang et al., 2003).

The pure thermal deformation of the crystal is obtained by assuming that the initial strain in the crystal is negligible and the crystal is flat. The displacement results are directly obtained from FEA for the silicon crystal under power load. The thermal slope along the meridional axis is calculated from the derivative of the displacement $U_{Z}$ (normal to the crystal surface) to the crystal coordinate $x_{\mathrm{c}}$ (slope $\left.=\partial U_{Z} / \partial x_{\mathrm{c}}\right)$. For each of these slope profiles within the footprint we calculate the corresponding RMS value. FEA results for the deformed crystal shapes in the footprint area (illuminated by the X-ray beam) are shown in Figs. $9(a)-9(d)$ as well as the thermal deformation displacement $U_{Z}$ for four representative cases selected from the ID06 beamline measurements. The aperture 


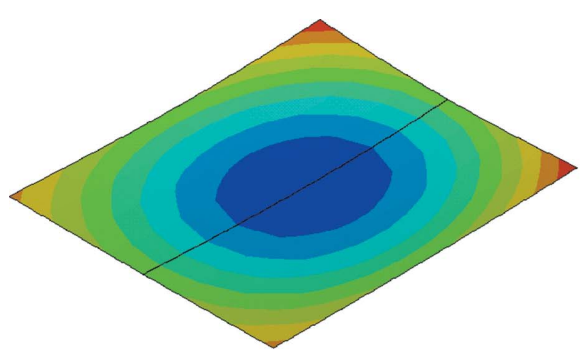

(a)

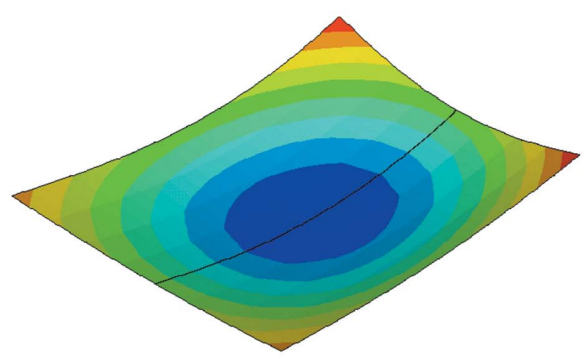

(b)

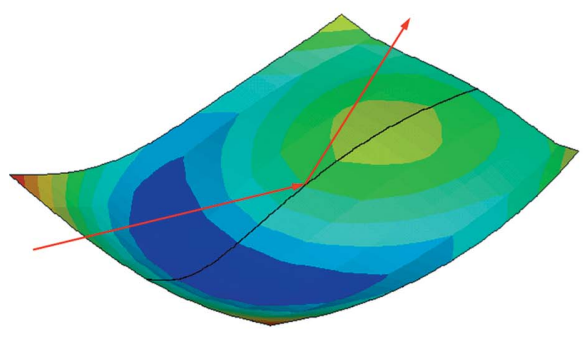

(c)

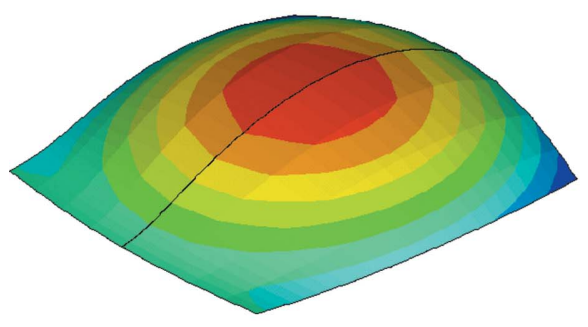

(d)

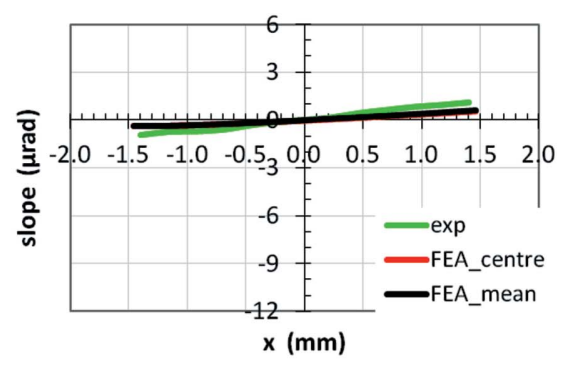

(e)

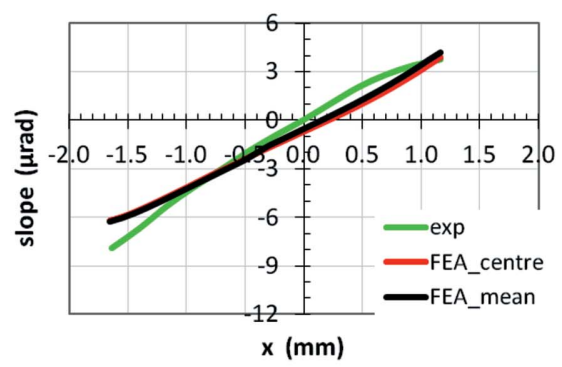

(f)

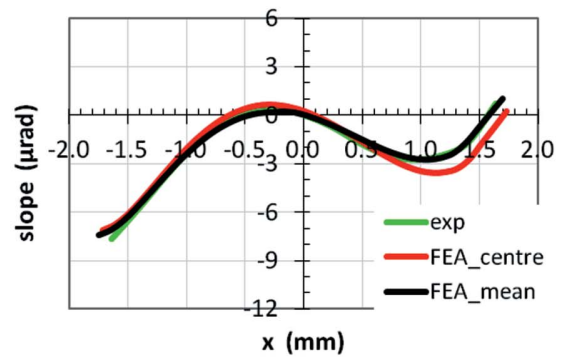

(g)

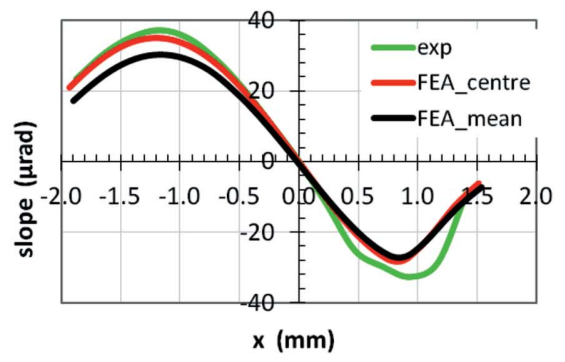

(h)

Figure 9

Left column $(a, b, c, d)$ : FEA results for the deformed crystal shapes in the footprint area with the thermal deformation displacement (the scale of the displacement is different for the four cases) in the direction normal to the crystal surface for four heat load conditions on the crystal at beamline ID06: $(a, e) P=19.1 \mathrm{~W}, T_{\max }=85.0 \mathrm{~K},(b, f) P=194.5 \mathrm{~W}, T_{\max }=120.3 \mathrm{~K},(c, g) P=288.9 \mathrm{~W}, T_{\max }=$ $153.6 \mathrm{~K},(d, h) P=385.2 \mathrm{~W}, T_{\max }=205.7 \mathrm{~K}$. The primary slits opening is $2 \mathrm{~mm} \times 1 \mathrm{~mm}(\mathrm{H} \times \mathrm{V})$. The red lines with arrows show the X-ray beam incident and reflecting directions. Right column $(e, f, g$, $h)$ : comparison of calculated and measured thermal slope distributions on the crystal surface in the meridional direction (red line: along the central axis; black line: averaged over the transverse footprint width) for the four heat load conditions. The same scales for the slope distribution are used in $(e),(f)$ and $(g)$; a much larger vertical scale is used for case $(h)$.

of the primary slits is $2 \mathrm{~mm} \times 1 \mathrm{~mm}(\mathrm{H} \times \mathrm{V})$. The electron beam current and power load for these four cases are given in Table 3, which also includes the calculated maximum temperature and RMS slope error along the central axis of the footprint ( $x$-axis). On increasing the electron beam current or power load on the crystal, $(a)$ at a low absorbed power
$(19.1 \mathrm{~W})$ the crystal is slightly deformed in a concave shape; $(b)$ at a medium absorbed power (194.5 W) the crystal is significantly deformed in a concave shape, the maximum temperature in the crystal is $120.3 \mathrm{~K}$, close to $125 \mathrm{~K}$ where the thermal expansion coefficient of silicon is zero, but the thermal strain is negative; $(c)$ at a higher absorbed power $(288.9 \mathrm{~W})$ the crystal has a shape inversion from concave to convex, the maximum temperature in the crystal is $153.6 \mathrm{~K}$, the RMS thermal slope error is $2.19 \mu \mathrm{rad}$, smaller than the $2.98 \mu \mathrm{rad}$ for case $(b) ;(d)$ at an even higher absorbed power $(385.2 \mathrm{~W})$ the crystal is very much deformed in a convex shape, the maximum temperature in the crystal is 205.7 K, the RMS thermal slope error is $22.03 \mu \mathrm{rad}$. The absorbed power in case $(d)$ is only $33 \%$ higher than in case (c), but the thermal deformation (RMS slope error) is increased by a factor of ten, a very strong non-linear effect. In case $(c)$ one observes that the shape inversion from concave to convex occurs on the incident side of the footprint at a smaller absorbed power than on the exit side. This can be explained by the power absorption in the inclined volume with beam incidence at Bragg angle $\theta_{\mathbf{B}}=25.4^{\circ}$ : the volume under the upstream half of the footprint absorbs less power than the volume under the downstream half of the footprint.

The thermal slope distribution from the FEA simulations are also compared with the measurement results for these four cases [Figs. 9(e)-9(h)]. The FEA results are given for the central axis (FEA_centre), and also averaged over the transverse footprint width (FEA_mean). The averaged slope profile is very close to (though slightly smaller than) the one along the central axis when the silicon crystal is concave (cases $e, f$ ), but significantly smaller when the crystal becomes convex (cases $g$ and $h$ ). As explained in $\$ 2.3 .2$, the measured slope profile of the crystal is a weighted average over the transverse footprint width, with highest weight coefficient along the central axis; the average of the FEA slope distribution should be made with the same weight function. But the latter is unknown. Therefore we use the simple average for FEA results which are in very good agreement with the experimental results in the first three cases $(e, f, g)$. Small discre- 
Table 3

Electron beam current $(I)$, total power absorbed by the silicon crystal $(P)$, calculated maximum temperature $\left(T_{\max }\right)$ and RMS slope error along the central axis of the footprint for the four cases shown in Fig. 9.

\begin{tabular}{lllcc}
\hline Case & $\begin{array}{l}\text { Electron beam } \\
\text { current } I(\mathrm{~mA})\end{array}$ & $\begin{array}{l}\text { Absorbed } \\
\text { power } P(\mathrm{~W})\end{array}$ & $T_{\max }(\mathrm{K})$ & $\begin{array}{l}\mathrm{RMS}_{\text {slope }} \\
(\mu \mathrm{rad})\end{array}$ \\
\hline$(a)$ & 9.9 & 19.1 & 85.0 & 0.30 \\
$(b)$ & 101 & 194.5 & 120.3 & 2.98 \\
$(c)$ & 150 & 288.9 & 153.6 & 2.19 \\
$(d)$ & 200 & 385.2 & 205.7 & 22.03 \\
\hline
\end{tabular}

pancies are found in case $(h)$, the case of high heat load and larger thermal deformation. The slope profile along the central axis from FEA is slightly larger than the experimental result in case $(g)$, but in good agreement in case $(h)$. The accuracy of the measurements of the crystal slope distribution is about $1 \mu \mathrm{rad}$. It should be emphasized that the slope distributions of the crystal in the four cases are very different, corresponding to crystal shapes changing from concave to convex, and the FEA simulations agree very well with the measurement results. This good agreement confirms the pertinence of the choice of parameters described in the previous section in FEA. The difference of the thermal slope distribution between experiments and FEA in cases $(e)$ and $(f)$ shows that the initial shape of the first crystal is concave with a slope error estimated at $1.1 \mu \mathrm{rad}$ peak-to-peak and about $0.45 \mu \mathrm{rad}$ RMS.

For a global picture of the thermally deformed silicon crystal at various heat loads, we have performed FEA simulations as presented above for many values of electron beam current, and plotted the thermal slope error in RMS versus absorbed power. For each power load condition, the RMS slope error is calculated from the two slope error profiles (FEA_centre, FEA_mean) described above, which covers the full footprint length defined by the vertical opening of the primary slits. For the ID06 beamline, this vertical opening is $1 \mathrm{~mm}$. The FWHM of the central cone undulator radiation is about $0.5 \mathrm{~mm}$ in the vertical at the position of the primary slits, which is half of the primary slit vertical opening. It is also interesting to calculate the RMS slope error over the length corresponding to the projection of the undulator central cone, which is approximately half a footprint length. The RMS thermal slope errors shown in Fig. 10 are calculated over the whole footprint or half footprint (_fp/2) length, from the slope distribution along the central axis or averaged (_av) over the transverse footprint width. The averaged RMS slope error is very close to the one along the central axis for power values less than $280 \mathrm{~W}$ (around the local minimum), but it differs significantly for higher power. The RMS slope calculated over the half footprint length is roughly half of that over the whole footprint length for power smaller than the one corresponding to the local minimum. The four points $(a, b, c, d)$ in Fig. 10 correspond to the four heat load conditions shown in Fig. 9. The position of these four points in Fig. 10 and the corresponding crystal shapes shown in Figs. $9(a)-9(d)$ give us a view of different working points within the global behaviour of the

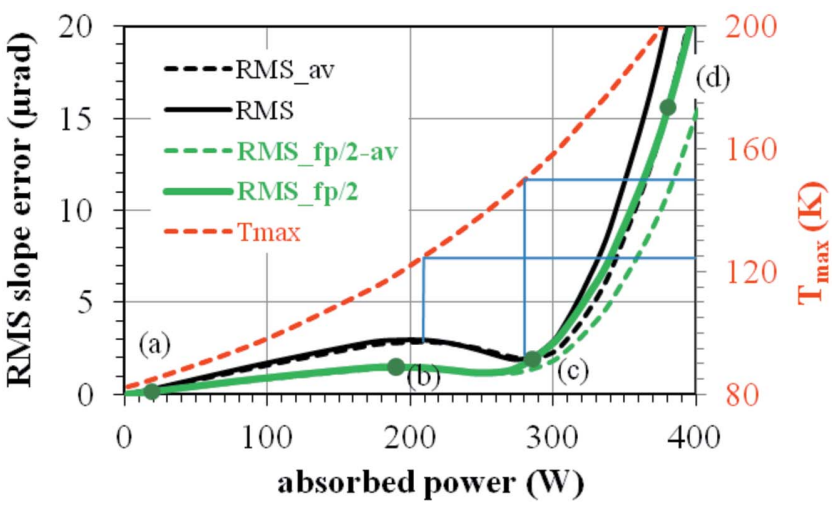

Figure 10

RMS thermal slope error and the maximum temperature of the ID06 LN2-cooled silicon crystal versus absorbed power. RMS values are calculated over the whole footprint or half footprint (_fp/2) length, from the slope distribution along the central axis or averaged (_av) over the transverse footprint width. The primary slits opening is $2 \mathrm{~mm} \times 1 \mathrm{~mm}$ $(\mathrm{H} \times \mathrm{V})$. The four points $(a, b, c, d)$ correspond to the four heat load conditions shown in Fig. 9.

RMS slope error and the corresponding deformed shape of the silicon crystal at various heat loads.

The maximum temperature of the crystal versus absorbed power is also shown in Fig. 10. It is about $125 \mathrm{~K}$ at the local maximum in the curve of the RMS slope error calculated over the whole footprint. This local maximum corresponds to the most concave shape of the silicon crystal with accumulated thermal contraction effects. Above $125 \mathrm{~K}$ the silicon crystal is in thermal expansion. The concave shape, as well as the RMS slope, is reduced to a local minimum in the curve of the slope versus the absorbed power. The maximum temperature of the crystal at this local minimum is about $150 \mathrm{~K}$, significantly higher than $125 \mathrm{~K}$ but smaller than $165 \mathrm{~K}$ (the theoretical uniform temperature for non-deformation, as discussed before).

We can also check our FEA results expressed in terms of RMS slope error against experimental results. It is shown in \$2.3.2 that the thermal slope error including the initial deformation of the crystal due to mounting is about $7.2 \mu \mathrm{rad}$ in FWHM (3.1 $\mu \mathrm{rad}$ RMS assuming Gaussian distributions) from the rocking curve width. The initial shape of the first crystal is concave with a slope error estimated to be $0.45 \mu \mathrm{rad}$ RMS (1.1 $\mu \mathrm{rad}$ peak-to-peak). The pure thermal slope error in RMS should be in the interval $(2.65,3.07) \mu \mathrm{rad}$, where the lower and upper limits correspond, respectively, to the cases where the thermal deformed shape and the initial shape of the crystal are perfectly correlated $(3.1-0.45=2.65 \mu \mathrm{rad})$ and uncorrelated $\left[\left(3.1^{2}-0.45^{2}\right)^{1 / 2}=3.07 \mu \mathrm{rad}\right]$. The experimental rocking curves were measured at a power load $P=194.5 \mathrm{~W}$ [point $(b)$ in Fig. 10]. The pure thermal RMS slope error from FEA is $2.98 \mu \mathrm{rad}$ along the central axis and $2.86 \mu \mathrm{rad}$ in a laterally averaged profile, calculated over the whole footprint length (Fig. 10). Therefore, the estimation of the thermal slope error from the rocking curve broadening measurements is consistent with the FEA results in RMS slope error calculated over the whole footprint length. 
After being validated by experiments, the FEA simulations can then be used for the design and optimization of beamline optics. An example is the LN2-cooled silicon crystals in the pre-monochromator for the ESRF UPBL6 beamline under construction. The first silicon crystal is a block $150 \mathrm{~mm}$ long, $60 \mathrm{~mm}$ wide and $100 \mathrm{~mm}$ thick, placed at $30 \mathrm{~m}$ from the U26 and U32 undulator sources. The photon energy scan range is 5-20 keV, corresponding to Bragg angles between $5.6^{\circ}$ and $23.1^{\circ}$. The beam size is defined by the aperture of the primary slits at $27 \mathrm{~m}: 1.8 \mathrm{~mm} \times 0.8 \mathrm{~mm}(\mathrm{H} \times \mathrm{V})$. The X-ray beam centre cone size is $1.2 \mathrm{~mm} \times 0.4 \mathrm{~mm}\left(\mathrm{FWHM}_{\mathrm{h}} \times \mathrm{FWHM}_{\mathrm{v}}\right)$. We concentrated on the FEA results in the area illuminated by the centre cone on the crystal surface. The RMS thermal slope error is plotted versus absorbed power at various Bragg angles in Fig. 11. The working points corresponding to an electron beam current of $200 \mathrm{~mA}$ (maximum operational current) are indicated in Fig. 11, and all sit on the flat part (less sensitive to the absorbed power) or left-hand side of the local minimum of the curves, where the silicon crystal has a concave shape. In this zone or at these working points the longitudinal profiles of the crystal are approximately circular and their RMS slope error (proportional to the inverse of the radius of curvature) increases linearly with power. The thermal deformations in this zone can be compensated by other focusing elements in the beamline. Regardless of the angle of incidence, the thermal slope error of the crystal can be kept under $2 \mu \mathrm{rad}$. Moreover, it can be lower than $1 \mu \mathrm{rad}$ if the local minima are chosen as working points with high power load and may be good choices for specific experiments. However, these positions are quite unstable: a small increase in power may result in very high thermal deformations.

The detailed shape of the thermally deformed silicon crystal significantly affects the beam wavefront downstream from the DCM. A plane incident wave can be turned into a converging (diverging) one when the crystal is thermally deformed in a concave (convex) shape. When the crystal shape evolves from concave to convex, the crystal curvature is not constant along the diffracting direction and affects the spatial extension and

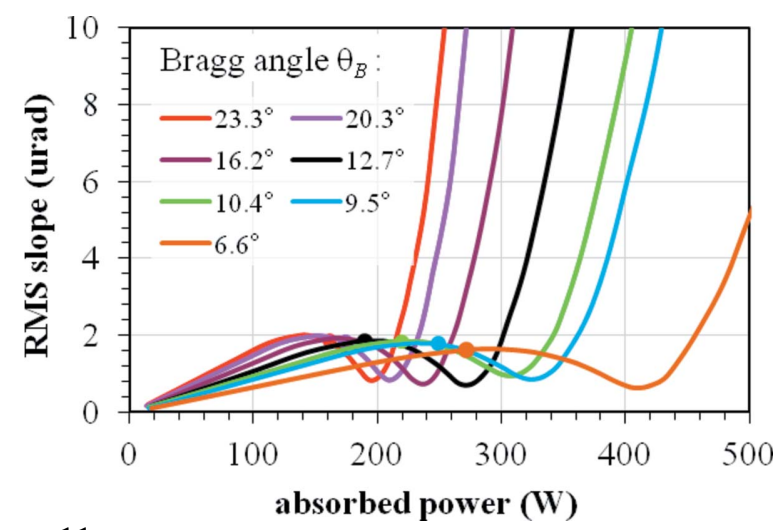

Figure 11

RMS thermal slope error of a LN2-cooled silicon crystal under variable heat load at different Bragg angles for the ESRF UPBL6 beamline project. The RMS thermal slope is calculated over the central cone illuminated length on the crystal surface (half footprint). Working points at $I=200 \mathrm{~mA}$ (the present most common operation electron beam current at the ESRF) are also shown as circle points.

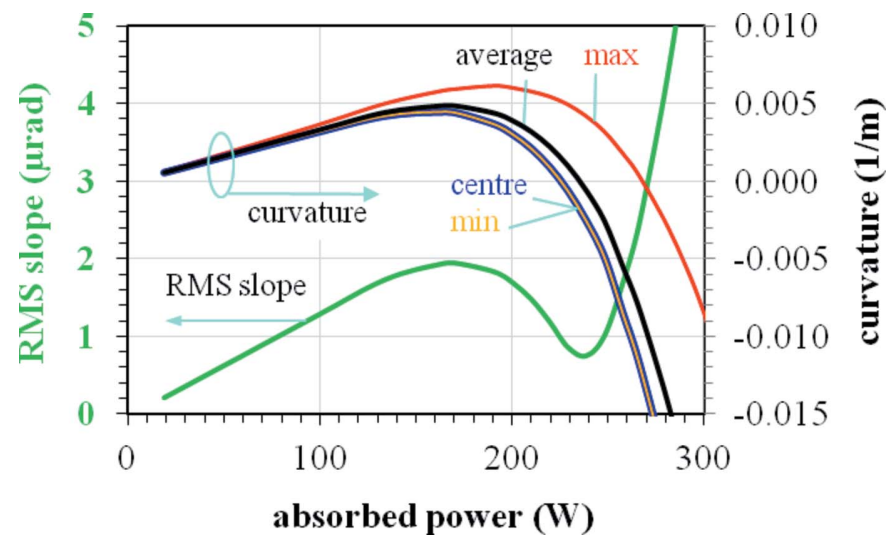

Figure 12

RMS thermal slope (green line, left axis) error versus absorbed power at Bragg angle $\theta_{\text {Bragg }}=16.2^{\circ}$ for the LN2-cooled silicon crystal for the ESRF UPBL06 beamline project. Different values of the (non-constant) curvature are plotted (right axis): at the centre of the footprint, the maximum, minimum and average over the central cone illuminated half footprint length. Note that in the absorbed power range $(225,270) \mathrm{W}$ the maximum curvature is positive (concave) whereas the minimum curvature is negative (convex).

shape of the wavefront. To estimate the non-uniformity of the curvature along the crystal, the RMS thermal slope error for Bragg angle $\theta_{\text {Bragg }}=16.2^{\circ}$ is plotted versus absorbed power (Fig. 12) together with the curvature at the centre of the footprint, and the maximum, minimum and average curvature over the crystal surface illuminated by the central cone. The average curvature is positive (concave shape) at a small power load, reaches a maximum and then goes through zero at about the same power loads as the local maximum and minimum in the curve of the RMS slope versus power, then becomes strongly negative (convex shape or bump) on increasing the absorbed power. The values of the curvature at the centre of the footprint are almost identical to those of the minimum curvature. However, it is worth pointing out that the difference between the maximum and minimum of the curvature indicates that the thermal deformed crystal shape is not spherical (or not constant curvature). Fig. 12 shows that the curvature induced on the monochromator by the thermal deformation is not uniform and may strongly modify the beam properties, with consequences that clearly depend on the specific beamline design and applications. The plots shown in Figs. 11 and 12 are a convenient guide for the design and optimization of the LN2-cooled silicon crystal monochromators, and help to define the beamline optical layout. The results in Fig. 11 also show that the heat load limit for the LN2-cooled silicon crystal varies strongly with the Bragg angle: $450 \mathrm{~W}$ for $\theta_{\mathrm{B}}=6.6^{\circ}, 230 \mathrm{~W}$ for $\theta_{\mathrm{B}}=23.1^{\circ}$. These heat load limits depend also on the beam size, but only slightly on the cooling coefficient as reported by Zhang et al. (2003).

\section{Summary and conclusions}

When measurements of diffracted intensity using a narrowgap exit slit at various vertical positions are combined with monochromator rocking angle scans, it is possible to measure 
the thermal deformation profile of LN2-cooled silicon crystals under heat load. This method is very simple, effective and easy to implement with existing beamline components. Up to now, the thermal deformation of the monochromator crystal has been indirectly studied by measuring the rocking curve width broadening, effect of the thermal distortion of the crystal. The method proposed here is a direct measurement of the thermal distortion of the crystal surface. The measurement accuracy depends mostly on the accuracy of the angular positioning and the heat load stability of the rotational stage of the second crystal, and also on the accuracy of the slits, and can be in the range of a fraction of a microradian.

The thermal deformation of LN2-cooled silicon crystal monochromators has been accurately predicted by FEA. The LN2-cooled silicon crystal surface is concave at low heat load, convex at high heat load, and in a complex shape, almost flat on average, at intermediate heat load. The FEA predictions are based on the correct use of material parameters in the FEA software, accurate estimation of the power load on the crystal and relevant boundary conditions including the power absorption and cooling parameters.

The excellent agreement between the measured crystal shapes and FEA simulations under different heat load conditions confirms, once more, the adequacy of using the finite-element modelling for the beamline design and optimization, and validates the use of FEA quantitative results, with a high level of reliability, to fulfil the particular and demanding conditions required by the ESRF Upgrade Programme.

\section{APPENDIX $A$ \\ ANSYS command for the temperature-dependent thermal expansion coefficient}

In the mathematical formulation used in the ANSYS code, the thermal strain is calculated as in equation (4) for constant thermal expansion coefficient $\alpha$, or using the secant coefficient of the thermal expansion as in equations (4) and (5). The input command is then 'MP, ALPX,...'. As the thermal expansion coefficient data in the literature are usually reported as a function of temperature (Touloukian et al., 1970b), the data should be first converted to the secant form by equation (6), then the command 'MP, ALPX, ...' can be used for data input.

Since the release of ANSYS 8.0, the instantaneous coefficient of the thermal expansion (as given in the literature) can be directly input in ANSYS with the command 'MP, CTEX, ...'. The data conversion according to equations (5) and (6) is then made within the ANSYS code. In both cases the reference temperature $T_{\text {ref }}$ is needed. For a LN2-cooled silicon monochromator crystal the reference temperature $T_{\text {ref }}$ should be the temperature of the cooled crystal before switching on the X-ray power ( $T_{\text {ref }}=77 \mathrm{~K}$ for instance). $A N S Y S$ uses $T_{\text {ref }}=$ 0 by default, therefore this parameter should always be carefully checked.

There was some confusion on how to input the data of the thermal expansion coefficient when using ANSYS code. Before ANSYS version 8 (released at the end of 2003), only the secant coefficient of the thermal expansion could be used with the command 'MP, ALPX,...'. Many ANSYS users (including ourselves) have directly input thermal expansion coefficient data from the literature using this command, thus obtaining an incorrect value of the temperature $(T=125 \mathrm{~K})$ at which the thermal deformation of the LN2-cooled silicon crystal reaches a local minimum. As a consequence the incorrect idea of thermal deformation being zero at $125 \mathrm{~K}$ had been widely spread in the synchrotron community. For silicon the thermal expansion coefficient is zero at $125 \mathrm{~K}$, but the thermal deformation of the LN2-cooled silicon crystal is not zero. The thermal strain of LN2-cooled silicon is zero at $165 \mathrm{~K}$ (Fig. $4 b$ in this paper). The X-ray power-induced thermal deformation reaches a local minimum at a maximum temperature of the crystal slightly lower than $165 \mathrm{~K}$. As an example, in the paper by Zhang et al. (2003) ANSYS version 6.1 was used and the thermal expansion coefficient from Touloukian et al. (1970b) was directly input with the only available ANSYS command 'MP, ALPX, ...'. We noticed this problem in 2006, and using the correct $A N S Y S$ command 'MP, CTEX,...' for the coefficient of thermal expansion we recalculated the results corresponding to Fig. 8 in the paper (Zhang et al., 2003), and reported as Fig. 13 here for the rocking curve widths (FWHM) as well as for the maximum temperature on the Si crystal versus total absorbed power. In Fig. 13 we added two more curves with respect to those appearing in Fig. 8 of that paper (Zhang et al., 2003): the recalculated rocking curve width FWHM and the maximum temperature of the crystal using the correctly input thermal expansion coefficient by the command 'MP, CTEX,...' and with an effective cooling coefficient of $1400 \mathrm{~W} \mathrm{~m}^{-2} \mathrm{~K}^{-1}$ (green lines). The recalculated rocking curve width FWHM with an effective cooling coefficient of $1400 \mathrm{~W} \mathrm{~m}^{-2} \mathrm{~K}^{-1}$ fit well with the experimental results reported in that paper. This implies that the effective cooling coefficient was $1400 \mathrm{~W} \mathrm{~m}^{-2} \mathrm{~K}^{-1}$

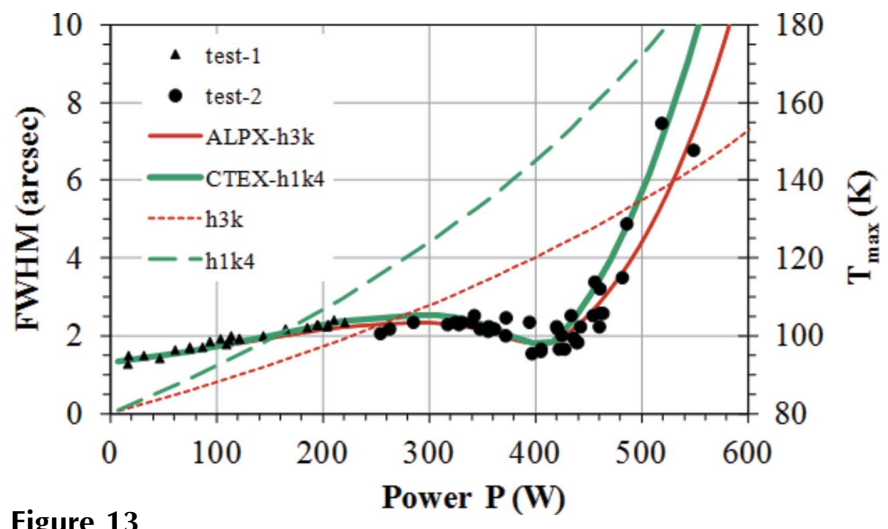

Figure 13

Rocking curve width FWHM and maximum temperature on a channelcut Si crystal as a function of the total absorbed power. Triangles and circles represent experimental data, the red line ALPX-h3k corresponds to previous calculated rocking curve widths FWHM with a cooling coefficient of $3000 \mathrm{~W} \mathrm{~m}^{-2} \mathrm{~K}^{-1}$ (Zhang et al., 2003), the green line CTEXh1k4 represents recalculated results with the coefficient of thermal expansion input by 'MP, CTEX, ...'. The corresponding maximum crystal temperature curves are the dashed red line $\mathrm{h} 3 \mathrm{k}$, and dashed green line $\mathrm{h} 1 \mathrm{k} 4$. Here $\mathrm{h} 1 \mathrm{k} 4$ and $\mathrm{h} 3 \mathrm{k}$ correspond to cooling coefficients of 1400 and $3000 \mathrm{~W} \mathrm{~m}^{-2} \mathrm{~K}^{-1}$. The green lines correspond to the corrected FEA results. 
instead of $3000 \mathrm{~W} \mathrm{~m}^{-2} \mathrm{~K}^{-1}$. Therefore, the results published previously are qualitatively correct, and can be further corrected using a smaller effective cooling coefficient. The recalculated results show that the local minimum of the calculated FWHM corresponds to a maximum crystal temperature of about $150 \mathrm{~K}$. This is very close to results reported in Figs. 9 and 10 of the present paper.

The ESRF Accelerator and Source Division is acknowledged for the collaboration in the heat load tests with electron beam current ramping. The authors are grateful to Katrine Wong and Philippa Gaget for proof-reading the document and English corrections.

\section{References}

Bilderback, D. H., Freund, A. K., Knapp, G. S. \& Mills, D. M. (2000). J. Synchrotron Rad. 7, 53-60.

Chubar, O. \& Elleaume, P. (1998). Proceedings of the Sixth European Particle Accelerator Conference (EPAC'98), pp. 1177-1179.

Chumakov, A., Rüffer, R., Leupold, O., Celse, J.-P., Martel, K., Rossat, M. \& Lee, W.-K. (2004). J. Synchrotron Rad. 11, 132-141.

Hoszowska, J., Mocella, V., Zhang, L., Migliore, J. S., Freund, A. \& Ferrero, C. (2001). Nucl. Instrum. Methods Phys. Res. A, 467-468, 631-634.

Lee, W.-K., Fernandez, P. \& Mills, D. M. (2000). J. Synchrotron Rad. 7, $12-17$.

Lee, W.-K., Fezzaa, K., Fernandez, P., Tajiri, G. \& Mills, D. M. (2001). J. Synchrotron Rad. 8, 22-25.

Lee, W. K., Mills, D. M., Assoufid, L., Blasdell, R. C., Fernandez, P., Rogers, C. S. \& Smither, R. K. (1995). Opt. Eng. 34, 418-425.

Marion, P., Zhang, L., Vallet, L. \& Lesourd, M. (2004). Proceedings of MEDSI 2004 - Mechanical Engineering Design of SR Equipment and Instrumentation 2004. ESRF, Grenoble, France.

Marot, G. (1995). Opt. Eng. 34, 426-431.

Marot, G., Rossat, M., Freund, A., Joksch, S., Kawata, H., Zhang, L., Ziegler, E., Berman, L., Chapman, D., Hastings, J. B. \& Iarocci, M. (1992). Rev. Sci. Instrum. 63, 477.
Mocella, V., Ferrero, C., Freund, A., Hoszowska, J., Zhang, L. \& Epelboin, Y. (2001). Nucl. Instrum. Methods Phys. Res. A, 467-468, 414-417.

Mocella, V., Lee, W.-K., Tajiri, G., Mills, D., Ferrero, C. \& Epelboin, Y. (2003). J. Appl. Cryst. 36, 129-136.

Mochizuki, T., Kohmura, Y., Awaji, A., Suzuki, Y., Baron, A., Tamasaku, K., Yabashi, M., Yamazaki, H. \& Ishikawa, T. (2001). Nucl. Instrum. Methods Phys. Res. A, 467-468, 647-649.

Rogers, C. S., Mills, D. M., Lee, W. K., Knapp, G. S., Holmberg, J., Freund, A., Wulff, M., Rossat, M., Hanfland, M. \& Yamaoka, H. (1995). Rev. Sci. Instrum. 66, 2494-2499.

Sánchez del Río, M. \& Dejus, R. J. (2011). Proc. SPIE, 8141, 814115. Secco, E. \& Sánchez del Río, M. (2011). Proc. SPIE, 8141, 81410Z.

Tajiri, G., Lee, W.-K., Fernandez, P., Mills, D. M., Assoufid, L. \& Amirouche, F. (2001). J. Synchrotron Rad. 8, 1140-1148.

Takagi, S. (1962). Acta Cryst. 15, 1311-1312.

Takagi, S. (1969). J. Phys. Soc. Jpn, 26, 1239-1253.

Tanaka, T. \& Kitamura, H. (2000). SPECTRA - Synchrotron Radiation Calculation Program. Insertion Device Group, SPring8, Hyogo, Japan.

Taupin, D. (1964). Bull. Soc. Fr. Minéral. Cristallogr. 87, 469-511.

Taupin, D. (1967). Acta Cryst. 23, 25-35.

Touloukian, Y. S., Kirby, R. K., Taylor, R. E. \& Lee, T. Y. R. (1970a). Thermophysical Properties of Matter, Vol. 13, Thermal Expansion, pp. 154-161. New York/Washington: IFI/Plenum.

Touloukian, Y. S., Powell, R. W., Ho, C. Y. \& Klemens, P. G. (1970b). Thermophysical Properties, Vol. 1, Thermal Conductivity - Metallic Elements and Alloys, pp. 326-339. New York/Washington: IFI/ Plenum.

Vallet, L. \& Zhang, L. (2003). Résistance thermique de contact. Internal report. ESRF, Grenoble, France.

Wortman, J. J. \& Evans, R. A. (1965). J. Appl. Phys. 36, 153.

Zhang, L. (1993). Proc. SPIE, 1997, 223-235.

Zhang, L. (2009). AIP Conf. Proc. 1234, 797-800.

Zhang, L. \& Biasci, J. C. (2005). Heatload tests on ID6, ESRF Annual Machine Workshop. ESRF, Grenoble, France.

Zhang, L., Hoszowska, J., Migliore, J. S., Mocella, V., Ferrero, C. \& Freund, A. (2001). Nucl. Instrum. Methods Phys. Res. A, 467-468, 409-413.

Zhang, L., Lee, W.-K., Wulff, M. \& Eybert, L. (2003). J. Synchrotron Rad. 10, 313-319. 